\title{
Der Beitrag der Migranten an die lokale Entwicklung in Lateinamerika
}

Claude Auroi

\section{OpenEdition}

1 Journals

Electronic version

URL: http://journals.openedition.org/sjep/333

ISSN: 1663-9677

Publisher

Institut de hautes études internationales et du développement

Printed version

Date of publication: 1 décembre 2008

Number of pages: 141-161

ISBN: 978-2-940415-08-3

ISSN: $1660-5926$

Electronic reference

Claude Auroi, «Der Beitrag der Migranten an die lokale Entwicklung in Lateinamerika »,

Schweizerisches Jahrbuch für Entwicklungspolitik [Online], 27-2 | 2008, Online erschienen am: 31 Mai

2010, abgerufen am 07 September 2020. URL : http://journals.openedition.org/sjep/333

(c) The Graduate Institute 


\title{
Der Beitrag der Migranten an die lokale Entwicklung in Lateinamerika
}

\author{
Claude Auroi*
}

\section{Einführung}

Seit etwa zehn Jahren haben die globalen Migrationsbewegungen an Bedeutung gewonnen. Heute, in der Ära der Globalisierung, betreffen diese Migrationsbewegungen praktisch alle Regionen der Welt. In Lateinamerika hat sich die Zahl der Migranten, die in die Vereinigten Staaten, in andere Länder des Kontinents und nach Europa ausgewandert sind, zwischen 1990 und 2000 mehr als verdoppelt.

$\mathrm{Zu}$ den wichtigsten sozioökonomischen Aspekten der Migrationsbewegungen zählen die Geldmittel und die Produkte, welche die Migranten ihren Familien senden. Mit der wachsenden Migration hat auch das Volumen der Mittelüberweisungen lateinamerikanischer Migranten beträchtlich zugenommen: seit rund zwanzig Jahren jährlich um $15 \%^{1}$. Die Überweisungen sind von 5,8 Milliarden Dollar im Jahr 1990 auf offiziell 42,4 Milliarden Dollar im Jahr 2005 angestiegen ${ }^{2}$.

Die internationalen Finanzorganisationen (Internationaler Währungsfonds, Weltbank, Interamerikanische Entwicklungsbank) und die Fachorganisationen (Internationale Organisation für Migration, Entwicklungsprogramm der Vereinten Nationen) haben sich seit dem Beginn des 21. Jahrhunderts mit der Frage der Mittelüberweisungen und mit deren makro- und mikroökonomischen Folgen befasst. Die heute vorliegenden globalen Studien ${ }^{3}$ vermitteln ein relativ genaues Bild der Auswirkungen der Überweisungen auf bestimmte ökonomische und soziale Parameter.

* Professor am Institut de hautes études internationales et du développement (IHEID), Genf.

1 Interamerikanische Entwicklungsbank (IDB), Remittance Flows to Latin America and the Caribbean, 2004, Washington, DC, IDB, 2004. Weltbank, Global Economic Prospects 2006: Economic Implications of Remittances and Migration, Press launch, mit Christopher Neal, François Bourguignon, Hans Timmer, Dilip Ratha, William Shaw, Washington, DC, Weltbank, 16. November 2005.

2 Bimal Gosh, Migrants' Remittances and Development: Myths, Rhetoric and Realities, Genf, Internationale Organisation für Migration (IOM), 2006, S. 12, Tabelle 1.

3 Siehe die folgenden Studien der Interamerikanischen Entwicklungsbank (vollständige Quellenangaben in der Bibliografie am Schluss des vorliegenden Artikels): Survey of Remittance Senders: United States to Latin America (2002), Remittance Senders and Receivers: Tracking the Transnational Channels (2003), Sending Money Home: Remittance Recipients in the Dominican Republic and Remittance Senders from the United States (2004), State by State Survey of Remittance Senders: United States to Latin America (2004), Remittance Flows to Latin America and the Caribbean (2004, op. cit.), Remittances 2005: Promoting Financial Democracy (2006), Enviando Dinero a Casa: Marcador de la Industria de Remesas (2006); sowie: Pablo Fajnzylber and J. Humberto López, Close to Home: The Development Impact of Remittances in Latin America, Konferenzausgabe, Washington, DC, Weltbank, 2007. 
Der vorliegende Artikel behandelt hauptsächlich die Mittelüberweisungen der Migranten sowie die lokale und regionale Wirtschaftsentwicklung in Lateinamerika. Als erstes sollen die makroökonomischen Auswirkungen auf die Zielländer der Überweisungen beleuchtet werden. Da die Mittelüberweisungen jedoch eine wesentliche „Mikro“-Dimension beinhalten - es geht in der Regel um private Gelder im Rahmen der Familie - wird anschliessend untersucht, wozu die Empfänger dieses Geld verwenden. Zahlreiche Studien belegen, dass die Familien von Migranten $75 \%$ bis $80 \%$ des Geldes für Nahrungsmittel, Bekleidung, Haushaltsausstattung und Gesundheit ausgeben. Werden die Bildungsausgaben mit berücksichtigt, so bleiben knapp $5 \%$ bis $10 \%$ für Ausgaben übrig, die als Investitionen definiert werden können, z.B. Landerwerb, Hauskauf oder Gründung von kleinen Läden und Firmen. Aus diesem Grund wurde häufig die Auffassung vertreten, dass die Mittelüberweisungen im Wesentlichen der Armutsbekämpfung dienten und den im Heimatland geblieben Familienangehörigen erlaubten, überhaupt zu überleben.

Einige Studien schlossen jedoch in die genauere Definition des Begriffs „Investition" auch die Dauergüter und die Ausbildung mit ein ${ }^{4}$. Auf dieser Basis wird hier geprüft, ob die von den transnationalen Migrantenfamilien verfolgten Ausgabenstrategien vereinbar sind mit ihren Zukunftsplänen.

Ein Schlüssel zum Verständnis der Veränderungen, welche sich aus der Migration ergeben, liegt sicherlich im engeren Rahmen der Gemeinschaften und Regionen mit ausgeprägter Emigration. Im Mittelpunkt stehen die Veränderungen in ländlichen Gebieten, weil die Auswirkungen des wachsenden Mittelzuflusses die Intensität der Bodennutzung, die Anbaupläne, Grundstückskäufe, das Arbeitskräfteangebot, die Arbeit der Frauen sowie die Landflucht und die Emigration in die Städte beeinflussen.

Sicherlich wirken sich die Migration und die Mittelüberweisungen der Migranten auch auf die soziopolitischen Strukturen der kommunalen Regierungsführung aus; in überwiegend autochthonen Gemeinschaften verändern sie das Verhältnis zwischen sozialen Gruppen und ethnischen Gruppen.

Angesichts der Vielfalt des Subkontinents wäre es anmassend, ausgehend von bestimmten Fällen, selbst wenn es Nationen sind, die Tendenzen auf ganz Lateinamerika zu extrapolieren. Jedes Land und jede Region besitzt Besonderheiten: wirtschaftlicher, institutioneller und finanzieller Kontext, Art der Migration und der Migranten, Art der Mittelüberweisung und Investitionsmöglichkeiten. Trotzdem gibt es bestimmte „Grundsätze“ zur Migration sowie zu den Geldsendungen und Sachleistungen. Für Lateinamerika sind dies die vorherrschende Migration in die Vereinigten Staaten (75\%) und Spanien, die regelmässigen Geldsendungen über viele Jahre hinweg und die beinahe klischeehaften Ausgabemuster und Sparverhalten (Investitionen), sofern letztere möglich sind.

Die Auswirkungen der Migration führen einen sozialen Wandel herbei, der als positiv bezeichnet werden kann, weil er die nationale und internationale Öffnung und den Initiativgeist stimuliert; daneben gibt es jedoch auch andere kontraproduktive und unerwünschte Auswirkungen oder „Nebenwirkungen“ der sozioökonomischen Veränderungen. Es geht dabei um die Fragen der Arbeits-

4 Pablo Fajnzylber and J. Humberto López, op. cit. Jim Airola, „The Use of Remittance Income in Mexico (Report)“, International Migration Review, Bd. 41, Nr. 4, 2007. 
intensität und der Beteiligung an der Arbeitswelt, die Preisschwankungen der Güter und Dienstleistungen, die Geschlechterbeziehungen in den Migrantenfamilien, die Ausbildung der Kinder, den verschwenderischen oder auf Prestige ausgerichteten Umgang mit Geld zur Stärkung des Sozialkapitals und um die Investitionsentscheidungen.

Unsere Analyse geht von der Perspektive der nachhaltigen Entwicklung der armen Migrantenfamilien und ihrer Gemeinschaften aus. Treffen die Familien mit Blick auf ihre Überlebensfähigkeit während und nach der Migration die richtigen Entscheidungen? Tragen die Beziehungen zwischen Migranten und Nicht-Migranten zu Fortschritten für die ganze Gemeinschaft bei oder führen sie zu einer Verschärfung der sozialen Ungleichheiten und sogar zur Ausgrenzung bestimmter Gruppen?

Lückenlose Antworten auf alle Facetten dieser Fragen sind angesichts der verfügbaren Fallstudien zur jüngeren Vergangenheit, aber auch wegen der Grenzen des vorliegenden Beitrags momentan nicht möglich. Deshalb werden im Wesentlichen zwei Arten von Quellen herangezogen: erstens die Studien und Kurzberichte zu früheren Studien (z.B. denjenigen der Interamerikanischen Entwicklungsbank oder der Weltbank), zweitens Studien mit einer Zusammenfassung bestimmter praktischer Fallbeispiele, besonders für Mexiko ${ }^{5}$ sowie einige jüngere Studien zu Gemeinschaften in der Dominikanischen Republik, Bolivien, Ecuador, Peru und Mexiko. Allerdings wäre es anmassend, den vorliegenden Artikel als Zusammenfassung der gesamten einschlägigen Fachliteratur auszugeben oder zu behaupten, dass wir über ausreichende Feldstudien, Dissertationen oder Diplomarbeiten verfügten, um erschöpfende Vergleiche anzustellen.

\section{Volumen der Geldflüsse und globale wirtschaftliche Bedeutung}

Im Jahr 2005 betrugen die Heimatüberweisungen der Migranten nach Lateinamerika laut offiziellen Angaben über 50 Milliarden Dollar ${ }^{6}$; hinzu kommen die Beträge, die weder von Banken noch von Geldüberweisungsbüros erfasst werden und die wahrscheinlich $50 \%$ des oben erwähnten Totalbetrags ausmachen. Dieser Beitrag spielt für die Volkswirtschaften der betroffenen Länder selbstverständlich eine wichtige Rolle. Der Betrag übertrifft die Summe aller Exporte und sogar den Gesamtwert der internationalen Hilfe und der Direktinvestitionen und trägt damit wesentlich zur Verringerung der Schuldenlast und zum Zahlungsbilanzausgleich bei.

5 Siehe: Jorge Durand and Douglas S. Massey, „Mexican Migration to the US: A Critical Review“, Latin American Research Review, Bd. 27, Nr. 2, 1992, S. 3-43. Fernando Lozano Ascencio, „De excluidos sociales a heroes sexenales. Discurso oficial y remesas en Mexico“, in Raúl Delgado Wise und Beatrice Knerr (coord.), Contribuciones al análisis de la migración internacional y el desarrollo regional en México, Universidad Autónoma de Zacatecas, 2005, S. 41-65.

6 Inter-American Dialogue (IAD), Making the Most of Family Remittances, Second Report of the InterAmerican Dialogue Task Force on Remittances, Washington, DC, IAD, 2007. Die Gremien der Interamerikanischen Entwicklungsbank (IDB) sprechen für 2005 von 42,4 Milliarden Dollar; dieser Betrag umfasst allerdings nur die von den staatlichen Zentralbanken verbuchten offiziellen Überweisungen. Die nicht verbuchten Geldüberweisungen werden auf 50\% des offiziellen Gesamtwertes geschätzt. Angesichts dieser Unsicherheitsmarge erscheint die Schätzung von 50 bis 60 Milliarden Dollar angemessen. 
Die Mittelüberweisungen machen einen variablen Anteil des Bruttoinlandsprodukts (BIP) aus; in Costa Rica sind es z.B. 1,8\% des BIP, in Mexiko 2,8\%, in Guyana 34,3\%, in Haiti 20,7\%, in der Dominikanischen Republik 9,1\%, in Bolivien 8,5\% und in Peru 3,2\%7.Der Vergleich des Werts der Geldüberweisungen mit dem Wert der Exporte, besonders der Agrarexporte, verdeutlicht den Stellenwert der Heimatüberweisungen in den unterschiedlichen Volkswirtschaften (Tabelle 1).

Tabelle 1: Vergleich der Mittelüberweisungen nach Land

\begin{tabular}{|c|c|c|c|c|c|c|}
\hline Land & $\begin{array}{c}\text { Wert der } \\
\text { Über- } \\
\text { weisungen } \\
\text { (Mio. Dollar) }\end{array}$ & $\begin{array}{l}\text { Bevöl- } \\
\text { kerung } \\
\text { (Mio.) }\end{array}$ & $\begin{array}{c}\text { Wert der } \\
\text { Überweisungen } \\
\text { pro Einwohner } \\
\text { (Dollar) }\end{array}$ & $\begin{array}{c}\text { Wert der } \\
\text { Über- } \\
\text { weisungen } \\
\text { in } \% \text { des BIP }\end{array}$ & $\begin{array}{c}\text { Wert der } \\
\text { Überweisungen } \\
\text { in \% der } \\
\text { Exporte }\end{array}$ & $\begin{array}{c}\text { Über- } \\
\text { weisungen } \\
\text { in \% der } \\
\text { Agrarexporte }\end{array}$ \\
\hline Belize & 81 & 0.27 & 300 & 9 & 23 & 64 \\
\hline Bolivien & 860 & 9.18 & 93.7 & 8.5 & 33 & 68 \\
\hline Costa Rica & 362 & 4.33 & 83.6 & 1.8 & 5 & 16 \\
\hline Domin. Rep. & 2682 & 8.4 & 319.3 & 9.1 & 45 & 377 \\
\hline Ecuador & 2005 & 13.23 & 151.5 & 6.4 & 20 & 89 \\
\hline El Salvador & 2830 & 6.88 & 411.3 & 17.1 & 80 & 596 \\
\hline Guatemala & 2993 & 12.6 & 237.5 & 9.3 & 77 & 189 \\
\hline Guyana & 270 & 0.75 & 360 & 34.3 & 49 & 75 \\
\hline Haití & 1077 & 8.53 & 126.7 & 20.7 & 224 & 5143 \\
\hline Honduras & 1763 & 7.2 & 244.9 & 21.2 & 69 & 138 \\
\hline Jamaika & 1651 & 2.65 & 623 & 19 & 104 & 562 \\
\hline Kolumbien & 4126 & 45.6 & 90.5 & 4.1 & 19 & 114 \\
\hline Mexiko & 20034 & 107.3 & 186.7 & 2.8 & 10 & 168 \\
\hline Nicaragua & 850 & 5.49 & 154.8 & 16.9 & 55 & 158 \\
\hline Peru & 2495 & 28 & 89.1 & 3.2 & 15 & 120 \\
\hline Venezuela & 272 & 26.75 & 10.2 & 0.2 & 0.5 & 123 \\
\hline
\end{tabular}

Quelle: Die Angaben stammen aus Inter-American Development Bank (IDB), Remittances 2005: Promoting Financial Democracy, Washintgon, DC, IDB, 2006, Zahlen 2004.

Daneben wurden die Geldüberweisungen in bestimmte Länder mit dem Wert der wichtigsten Exportgüter dieser Länder verglichen ${ }^{8}$. Das wichtigste „Exportpro-

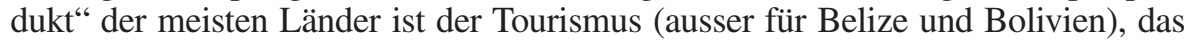
zweitwichtigste ein Agrar-, Bergbau- oder Kohlenstoffprodukt. Der Gesamtwert der Geldüberweisungen liegt durchweg deutlich über dem Wert des wichtigsten Exportproduktes (Ausnahmen sind Bolivien, Costa Rica, die Dominikanische Republik und Venezuela). Dies gilt auch für das zweitwichtigste Exportprodukt bzw. sogar für die Gesamtheit der Agrarausfuhren (siehe letzte Spalte von Tabelle 1). Die Geldüberweisungen sind allerdings nicht für die gleichen Empfänger bestimmt wie die Agrarexport- oder Tourismuserlöse; in der Regel kommen sie direkt den armen Familien zugute, während die Einnahmen aus der Rohstoffausfuhr bzw. aus dem Fremdenverkehr von Grosskonzernen einkassiert und nur ein kleiner Teil als Lohn an Landwirtschaftsarbeiter oder Kleinbauern umverteilt wird. Der Rest bildet eine enorme Kapitalmasse, die wieder investiert werden kann; für die persönlichen Überweisungen der Migranten ist dies nicht der Fall.

7 Weltbank, The Development Impact of Workers' Remittances in Latin America, Bericht, Nr. 37026, Washington, DC, Weltbank, 2006.

8 Claude Auroi, „Les envois de fonds pour le développement local. Réflexions à partir de cas latinoaméricains“, in Isabel Yépez und Gioconda Herrera (dir.), Nouvelles migrations latino-américaines en Europe, Barcelona, Publicacions i Edicions de la Universitat de Barcelona, S. 169-194. 
Überweisungen bestehen im Wesentlichen darin, dass ein Teil des Einkommens einer ,transnationalen Familie“" an die übrigen Familienmitglieder geschickt wird, die in einem anderen Land leben als demjenigen, in dem der Lohn erwirtschaftet wurde. Diese Einkommen werden nicht mit anderen ähnlichen Einkommen auf einem Bankkonto vermischt, sondern von der Familie rasch verbraucht. Deshalb ist die Frage wichtig, welche Beträge die Familien pro Monat und pro Jahr erhalten und welches die unterschiedlichen Verwendungszwecke sind. Dies gibt Aufschluss darüber, ob die Ausgaben eher für den täglichen Verbrauch oder/und für weitere produktive Zwecke dienen.

\section{Geldüberweisungen bei den Familien}

Die Geldüberweisungen der Migranten variieren in puncto Gesamtvolumen und Anteil pro Einwohner je nach Land erheblich. Mexiko ist mit jährlich 18 Milliarden Dollar laut den offiziellen Zahlen weltweit Spitzenreiter der Empfängerländer; pro Einwohner gerechnet führt jedoch Jamaika mit insgesamt jährlich 600 Dollar die Liste an. In Haiti und Honduras machen die Überweisungen den grössten Prozentsatz des BIP aus (über 20\%) ${ }^{10}$. Allgemein verzeichnet die Region Karibik und Zentralamerika die höchsten Prozente an Überweisungen, was u.a. auf den höchsten Prozentsatz an Migranten in die Vereinigten Staaten und in zweiter Linie nach Europa zurückgeht. Der „Braindrain“ trägt stark zu den Finanzströmen bei, denn mit zunehmendem Ausbildungsniveau steigen die Aussichten auf einen besser bezahlten Job im Gastland. „Über 80\% der Mittelschulabsolventen in Ländern wie Haiti, Jamaika, Grenada oder Guyana leben im Ausland, die meisten davon in den Vereinigten Staaten" "11. Man könnte beinahe sagen, dass die Inseln und die kleinen Karibikstaaten einen Teil des nordamerikanischen Komplexes bilden, für den das Symbol NAFTA ${ }^{12}$ steht.

Je südlicher das Land, desto geringer die Überweisungsbeträge und desto niedriger die Emigrationsrate. In Venezuela, Chile und Uruguay sind die Überweisungen praktisch nicht vorhanden bzw. sehr gering; in Surinam und in ganz Südamerika sind sie verglichen mit Zentralamerika bescheiden. Allerdings bedeutet dies nicht, dass diese Beträge - als BIP-Anteil oder per capita ausgedrückt - zu vernachlässigen sind, denn man muss auch die Empfänger der Überweisungen kennen. Kommen sie eher Familien mit mittlerem Einkommen oder armen Familien zugute? Wie erfolgt die Aufteilung, und in welchen spezifischen Regionen?

9 Manuel Orozco hat diesen Begriff geprägt und populär gemacht. Bisweilen wird auch der Ausdruck ,internationale Gemeinschaft“ verwendet, der besonders für Dörfer oder Stadtviertel mit hohem Migrationsanteil relevant ist. Manuel Orozco, Transnational Families: Lives on the Edge, but in Pursuit of Change, 2006, zitiert von Ezra Rosser, Remittances, College of Law Research Paper, Nr. 40, Washington, DC, American University College of Law, 2008, <http://ssrn.com/abstract=1024177>, S. 11 und Anmerkung 41.

10 Weltbank, The Development Impact of Workers'Remittances in Latin America, op. cit.

11 Pablo Fajnzylber und J. Humberto López, op. cit., S. 11 (Anm. d. $\ddot{U}$. : aus der französischen Übersetzung).

12 NAFTA: Nordamerikanisches Freihandelsabkommen zwischen Kanada, den Vereinigten Staaten und Mexiko. CARICOM: Karibischer Binnenmarkt. Der wichtigste Aus- und Einfuhrpartner der CARICOM-Mitglieder und Mexikos sind die Vereinigten Staaten. 
Einigen Studien zufolge bestehen die Überweisungen aus durchschnittlich 200 bis 300 Dollar mehrmals jährlich oder sogar jeden Monat ${ }^{13}$. Die Beträge variieren je nach Gastland, Beschäftigung und Lebenshaltungskosten des Migranten. 2004 umfassten die Überweisungen in die Dominikanische Republik durchschnittlich 150 Dollar mit 12 bis 15 jährlichen Sendungen ${ }^{14}$.

Der Migrant, der diese Gelder erwirtschaftet, überweist damit rund 10\% seines Verdienstes im Gastland (bei nur vorübergehendem Aufenthalt bisweilen sogar noch mehr). Dies mag bescheiden erscheinen; Migranten verdienen jedoch nur 1500 bis 2000 Dollar. Überdies müssen die Arbeitnehmer auch ihre Miete bezahlen und manchmal eines oder mehrere Familienmitglieder vor Ort unterhalten. Bisweilen investieren sie in eine Wohnung in den Vereinigten Staaten oder legen Bargeld auf die hohe Kante, um sich selbst und besonders die Familie im Heimatland gegen „Schicksalsschläge“ zu schützen. Die regelmässigen Bargeldsendungen werden durch ausserordentliche Überweisungen ergänzt, z.B. für den Bau eines Hauses, für das Schulgeld oder für bestimmte Feiern. Der Überweisungsrhythmus für die Dominikanische Republik weist im November-Dezember ein Hoch und im März ein Tief aus ; die Differenz beträgt beinahe 50\%. ${ }^{15}$

\section{Überweisungsmechanismen und Kontrolle der Geldströme}

Raúl Hernandez-Coss teilt die Geldflüsse in drei Etappen oder miles auf:

1. Die erste mile betrifft die Motivation, die den Migranten im Gastland anspornt, Geld zu senden. Diese Frage wird im vorliegenden Artikel nicht behandelt.

2. Die dazwischen liegende mile betrifft die eigentliche Überweisung, d.h. die physische oder virtuelle Bewegung des durch Arbeit oder auf sonstige Weise erwirtschafteten Geldes auf unterschiedlichem - institutionellem oder informellem - Weg.

3. Die letzte mile schliesslich betrifft die Verwendung des Geldes in den Herkunftsländern durch den Migranten oder seine Familie ${ }^{16}$.

Im vorliegenden Artikel zählt vor allem letzte mile, weil sie direkt den Verbrauch oder die Investition der Geldüberweisungen berührt. Die dazwischen liegende mile wird jedoch auch betrachtet: Dazu gehören nämlich die variablen Überweisungskosten, die vom Endbetrag, den er Empfänger erhält, abgezogen werden müssen.

Die internationalen und nationalen Finanzorganisationen und die Regierungen widmen derzeit der Zwischenmile das Hauptaugenmerk. Die Heimatüberweisungen der Migranten sind schlecht bekannt; informelle Sendungen machen Schätzungen zufolge die Hälfte der offiziellen Überweisungen aus. Offizielle

13 Weltbank, The Development Impact of Workers'Remittances in Latin America, op. cit.

14 Bendixen and Associates, Remittances and the Dominican Republic: Survey of Recipients in the Dominican Republic, Survey of Senders in the United States, November 2004, <http://www.iadb.org/ NEWS/docs/SurveysRemittanceFlows_e.ppt>.

15 Leonora Suki, Financial Institutions and the Remittance Market in the Domican Republic, New York, Center on Globalization and Sustainable Development, The Earth Institute at Columbia University, 2004, Tabelle 3 (Quelle: Zentralbank der Dominikanischen Republik), S. 12.

16 Raúl Hernandez-Coss, Lessons from the US-Mexico Remittance Corridor on Shifting from Informal to Formal Transfer System, World Bank Working Paper, Nr. 47, Washington, DC, Weltbank, 2005. 
Überweisungen werden wenn überhaupt nur geringfügig besteuert, was den Staat um Einnahmen bringt. Schliesslich fallen ziemlich hohe Überweisungsgebühren an (rund 10\%), welche die Beträge, die die Migrantenfamilien erhalten, reduzieren und die direkte Übergabe in die Hände der Empfänger attraktiver erscheinen lassen. Die Überweisungskosten variieren je nach Betrag; für kleinere Beträge (50 Dollar) sind sie höher ${ }^{17}$. Überweisungen umfassen durchschnittlich 150 bis 300 Dollar (mehrmals jährlich oder sogar monatlich); Gebühren von 8\% bis $9 \%$ (einschliesslich der Wechselkursdifferenzen) wirken deshalb abschreckend und führen dazu, dass Migranten nicht deklarierte Transfers vorziehen ${ }^{18}$ Eine vermehrte Institutionalisierung der Transfers über Banken hätte dagegen zur Folge, dass das Geld länger auf den Konten liegt, dass die Ersparnisse anwachsen und dass die Überweisungen häufiger für grössere Investitionen genutzt werden. Dazu müssten jedoch viel mehr Migranten überhaupt ein Bankkonto eröffnen. Gegenwärtig besitzen nur 25\% der Beteiligten an den beiden Enden des Übermittlungskanals ein Bankkonto ${ }^{19}$. Der Zugang zu einem ordentlichen Bankkonto wird durch verschiedene Faktoren eingeschränkt: staatliche Vorschriften zur Kontrolle der Illegalität und des Terrorismus (Patriot Act der Vereinigten Staaten), Bankgebühren, Wechselkurse, Misstrauen gegenüber dem System und Unwissen der Deponenten (häufig Analphabeten), Befürchtung, dass die Gelder eingefroren und besteuert werden. Obwohl 2004 die lateinamerikanischen Staaten und im gleichen Jahr auch die G-7 Leitlinien zur Förderung und Erleichterung der institutionellen Transfers erlassen haben ${ }^{20}$, ist nicht mit einer raschen Verbesserung der Lage zu rechnen. Die lateinamerikanischen Staaten erwägen fiskalische Vorschriften (Bolivien z.B. wollte die Heimatüberweisungen besteuern), welche zu einer Verschlimmerung führen dürften.

Feldstudien zeigen, dass es bestimmte Gepflogenheiten hinsichtlich der Geldüberweisungen gibt. Bolivianer, die provisorisch nach Argentinien emigrieren, bringen das Geld lieber direkt in der Tasche mit und kehren auch oft reich beladen mit Haushaltsutensilien und -geräten zurück. Wenn sie nicht zurückkehren, übergeben sie das Geld und die Sachgüter an paisanos, d.h. Dorfbewohner, denen sie vertrauen. Überweisungen über das offizielle System kommen selten $v{ }^{21}{ }^{2}$. In anderen Fällen - eher in Städten - werden die Transfers überwiegend auf dem Bankweg durchgeführt; rund zwei Drittel der Überweisungen zwischen Turin (Italien) und Quillabamba (Süden Perus) passieren das Bankensystem ${ }^{22}$. Die unterschiedlichen Präferenzen in beiden Beispielen erklären sich weitgehend aus der Entfernung und Häufigkeit der Rückreise, aber auch aus dem Umfang des Bankennetzes und der Bankdienstleistungen wie z.B. Kredite.

Wegen der geografischen Nähe von Argentinien und dem Süden Boliviens können Sachgüter importiert werden, z.B. Haushaltsgeräte, Fernseher und DVD-

17 Für die Dominikanische Republik z.B. betrugen die Überweisungsgebühren 2004 bei MoneyGram, Mateo Express und Pronto Envio für 50 Dollar 15\%, für 150 Dollar aber nur 8,33\% (bei Western Union waren es 19,98\% resp. 6,66\%). Leonora Suki, op. cit., Tabelle 10, S. 24.

18 Weltbank, The Development Impact of Workers' Remittances in Latin America, op. cit.

19 Pablo Fajnzylber and J. Humberto López, op. cit., S. 56.

20 Ibid., S. 50-51.

21 Roxana Dulón, Migración transnacional de bolivianos y bolivianas a la Argentina y su impacto en comunidades de origen, Sucre, Fundación Pasos y Grupo Chorlavi (Fondo Mink'a), 2008, S. 35.

22 Alejandrino S. Loaiza, L. Herrea y César Sotomayor, Las Remesas de los Migrantes Quillabambinos en Italia y sus Implicancias en el Desarrollo Local, Cusco, Proyecto de Desarrollo Corredor PunoCusco y Grupo Chorlavi, 2008, S. 4. 
Player (diese lassen sich per Flugzeug schwieriger befördern). Die Sachleistungen werden in den Studien im Allgemeinen nicht erfasst, leisten aber sicherlich einen wichtigen Beitrag. Die von Roxana Dulón untersuchten drei Dörfer erhalten jährlich mehr direkte Sachleistungen als Barbeträge; deren Höhe und monetäre Verwendungsmöglichkeiten gehen entsprechend zurück ${ }^{23}$.

\section{Verwendung der Mittel}

In der Folge werden einige Aspekte der Mittelverwendung in den Herkunftsgemeinschaften untersucht.

Als erstes ist festzustellen, dass nicht alle vom Geldsegen profitieren. In Mexiko, einem ausgeprägten Emigrationsland, erhalten nur 4,35\% der Haushalte Heimatüberweisungen ${ }^{24}$. Aber laut derselben Quelle beträgt der Prozentteil der Empfängerhaushalte in mexikanischen Bundesstaaten wie Zacatecas $13 \%$, in Michoacan 11\%; in den übrigen Bundesstaaten liegt der Anteil unter $10 \%$. Zwischen den ländlichen und städtischen Gegenden ist ein Unterschied festzustellen (rund 10\% bzw. 4\%).

Die lokalen empirischen Studien betreffen vor allem den Bundesstaat Zacatecas in Mexiko, eine Region mit langjähriger Auswanderungstradition in die Vereinigten Staaten ${ }^{25}$; in den übrigen Ländern hat die starke Migrationstradition erst in den 90er Jahren begonnen, so dass nur wenig Material vorliegt. Jüngere systematische Studien der Chorlavi-Gruppe bieten jedoch solide Daten für Kolumbien, Ecuador, Peru und Bolivien, allerdings immer in begrenzter Anzahl ${ }^{26}$. Diese Studien betreffen im Wesentlichen den ländlichen Raum. Deshalb werden wir uns darauf konzentrieren.

Als erstes ist festzustellen, dass die Verwendung der Heimatüberweisungen der Migranten in allen Regionen und Ländern grosse Ähnlichkeit aufweist. Das Gros der Mittel (ein gutes Drittel) dient zur Armutsbekämpfung, d.h. Ausgaben für Nahrung, Kleider und Unterkunft. An zweiter Stelle folgen Gesundheitsausgaben (zwischen 20\% und 25\%) und Ausbildungsausgaben für die Kinder (etwas niedrigerer Anteil). Zusammengerechnet machen diese drei Posten $80 \%$ der empfangenen Überweisungen aus; bestimmte Wirtschaftswissenschaftler und Analysten leiten daraus ab, dass es sich im Wesentlichen um Verbrauchsabgaben handelt. Bestimmte Haushaltsausgaben können jedoch als Investitionen betrachtet werden. Jim Airola zeigt für den Fall Mexikos ${ }^{27}$, dass die vergäng-

23 Roxana Dulón, op. cit.

24 Claudia Meza Merlos und Humberto Márquez Covarrubias, „Cambios en el patrón migratorio y pobreza en Zacatecas“, in Raúl Delgado Wise und Beatrice Knerr (coord.), op. cit., S. 251 Tabelle 3, gemäss der 12. Volkszählung (2000) in Mexiko.

25 Rodolfo Garcia Zamora, Migración, remesas y desarrollo, los retos de las organizaciones migrantes mexicanas en Estados Unidos, Mexico, Universidad Autónoma de Zacatecas, 2005. Volker Hamann, The Impact of International Labor Migration on Regional Development: The Example of Zacatecas, Mexiko, Kassel; Kassel University Press, 2006. Raúl Delgado Wise und Beatrice Knerr (coord.), op. cit.

26 Bei der Chorlavi-Gruppe handelt es sich um eine nichtstaatliche Forschungs- und Aktionsgruppe für Kleinbauern mit Sitz in Santiago, Chile. Die Gruppe hat vertiefte Studien zu den Veränderungen in der Landwirtschaft der Anden und zur Migration durchgeführt. Sie setzt sich für eine ökologische Landwirtschaft im Nahbereich ein. Vgl. <http://www.grupochorlavi.org>.

27 Jim Airola, op. cit. 
lichen Haushaltsgüter von den dauerhaften Haushaltsgütern (wie z.B. Haushaltgeräte) unterschieden werden müssen; letztere sind (ausgenommen Fernsehen und Radio) als Investitionsgüter einzustufen. Gestützt auf die Haushaltserhebung von 1984 bis $2000^{28}$ stellt der Autor fest, dass Migrantenfamilien andere Verbrauchsmuster aufweisen als Nicht-Migrantenfamilien: Sie investieren mehr in dauerhafte Gebrauchsgüter $(+56 \%)$ und geben mehr für die Gesundheit $(+44 \%)$ und für den Haushalt $(+17 \%)$ aus. In puncto Ausbildungsausgaben dagegen lässt die Erhebung keine wesentlichen Unterschiede nach Kategorien ersehen; betreffend die Nahrungsmittelausgaben ist bei den Migrantenfamilien eine relative Baisse festzustellen $(-8 \%)^{29}$.

Bei Gesundheitsausgaben handelt es sich teilweise um Humaninvestitionsausgaben, z.B. Prävention, Impfung der Kinder usw. Die Ausgaben für die Ausbildung, die ja zur Bildung von Humankapital führen, stellen ebenfalls eine Investition dar. Familien auf dem Land und in der Stadt wünschen sich gute Schulen für ihre Kinder, sogar wenn der Nachwuchs in eine andere Ortschaft geschickt werden muss oder die ganze Familie deswegen umzieht ${ }^{30}$. Zahlreiche Untersuchungen zu ländlichen Bewohnern in den Anden zeigen, dass die Bauern vor allem Geld sparen, um den Kindern eine bessere Zukunft zu ermöglichen, aber für sich selbst nicht an wesentliche Fortschritte glauben ${ }^{31}$.

Die übrigen $20 \%$ verteilen sich je nach Region unterschiedlich; am wichtigsten sind konkrete Ausbauarbeiten, der Erwerb eines neuen Hauses oder Grundstücks, die Tilgung von Schulden und sowie Bankersparnisse (zwischen 3\% bis 5\%).

\section{Finanzinvestitionen}

Der geringe Anteil (5\%), welcher den Bankspareinlagen gewidmet ist, bestärkt allgemein die Hypothese, wonach die Migranten und die Finanzinstitutionen kaum auf Ersparnisse und damit Investitionen setzen. Migranten zeigen eine deutliche „Präferenz für flüssige Mittel“ und beanspruchen das Bankensystem, oft wegen ihres illegalen Aufenthalts im Gastland, nur ungern. Allerdings zeigen die Studien, dass die individuellen Bankeinlagen punktuell grössere Beträge umfassen, d.h. rund 1000 Dollar oder mehr ${ }^{32}$, was für ärmere Schichten ein beträchtliches Kapital darstellt. Migranten nehmen häufig ausserordentliche Geldsendungen in Höhe von mehreren Tausend Dollar vor. Auch der Kauf eines Grundstücks oder der Hausbau stellt eine Investition dar.

28 Instituto Nacional de Estadisticas, Geografia e Informatica (INEGI), Encuesta Nacional de Ingresos y Gastos de Hogares, Mexiko, 2000.

29 Die Verbrauchsausgaben nehmen auch in absoluten Zahlen zu, jedoch in geringerem Umfang.

30 Geneviève Cortes, Partir pour rester: survie et mutation des sociétés paysannes (Bolivie), coll. „A travers champs“, Paris, Institut de recherche sur le développement (IRD), 2000. Gloria Camacho und Kattya Hernández, Territorios entrelazados: Impactos de la emigracion internacional para el desarrollo rural de Suscal, Quito, Instituto de estudios ecuatorianos, Grupo Chorlavi, 2008.

31 Claude Auroi, „Les agricultures andines, une lente évolution“, in Claude Auroi und Jean-Luc Maurer, Tradition et modernisation des économies rurales: Asie-Afrique-Amérique latine. Mélanges en l'honneur de Gilbert Etienne, Paris, Presses Universitaires de France, 1998, S. 285-309. Claude Auroi, „Situación alimenticia en América Latina y futuro de la producción agropecuaria para pequeños campesinos“, Colloque CEISAL, April 2007, Brüssel (erscheint 2008).

32 Gloria Camacho und Kattya Hernández, op. cit. 
Welchen Kriterien entspricht die Aufteilung der Transfers? Wie oben festgestellt liegt die physische Konsolidierung der Familien den Verhaltensweisen zu Grunde: Ausgaben für Haushalt, Gesundheit, für die Kinder und für höheren Komfort.

Ausgaben für die Wertvermehrung betreffen den Erwerb von physischem Kapital (Land, Grundstücke, Tiere, Bau von Brunnen und Bewässerungsleitungen, Kauf von Fahrzeugen usw. ${ }^{33}$ ), aber auch die bessere Schulbildung der Kinder.

Ein Teil der Ausgaben schliesslich betrifft die Erhaltung oder Vermehrung des sozialen Kapitals der Gemeinschaften: Es handelt sich um punktuelle Beiträge an Werke oder Bauten, z.B. Kirchen, Schulen, Jahresfeste und Geschenke ${ }^{34}$.

Angesichts der relativ inflexiblen sozialen Machtstrukturen in den ländlichen Gemeinschaften Lateinamerikas erscheint diese Strategie schlüssig und sinnvoll. Sie entspricht dem momentanen Bedarf (die eigene Armut bekämpfen, seine Identität behaupten) und dem künftigen Bedarf (für das Alter und für die Zukunft der nächsten Generationen vorsorgen). Der Umverteilungsprozess von Teilen der erworbenen Ressourcen an die Gemeinschaft verschafft Sozialprestige, das nicht unbedingt angestrebt wird, aber durchaus nützlich ist. Es ist nämlich wesentlich, den Anschluss an die Gemeinschaft zu bewahren, weil die Migration eines Tages endet und die Rückkehr ansteht.

Ist diese Strategie nachhaltig? Bei der Antwort auf diese Frage ist zu prüfen, ob die Migration per se nachhaltig ist: Um die Situation einer armen Familie vor Ort zu verbessern, müssen die Migranten offensichtlich viele Jahre lang Geld überweisen. Bei jungen Migranten (zwischen 20 und 40 Jahren) dürften sich die Transfers während gut einer Generation in der derzeitigen Form fortsetzen; deshalb handelt es sich um eine dauerhafte wirtschaftliche Investition.

\section{Bemühungen zur Umlenkung der Migrantengelder in kollektive Investitionen}

Miguel Moctezuma Longoria unterscheidet fünf Arten von Migranten-Investoren: kollektive Migranten, Unternehmer-Migranten, Sparer-Migranten, Migranten am Rand des Existenzminimums und ins Heimatland zurückgekehrte Migranten im Ruhestand ${ }^{35}$.

Im vorliegenden Artikel steht der kollektive Migrant im Mittelpunkt (er wird bisweilen mit dem Unternehmer-Migranten verwechselt). Letztere haben es in der Regel geschafft, im Gastland ein Geschäft zu gründen, z.B. ein Restaurant, einen Laden mit Produkten aus dem Herkunftsland oder einen Kleiderladen. Der dritte Migrantentypus spart, um sich ein Haus oder ein Grundstück zu kaufen. Der Migrant am Rande des Existenzminimums hat kein Geld für Investitionen zur Verfügung. Der Rentner-Migrant öffnet manchmal ein kleines Geschäft oder führt einen Kiosk.

33 Rodolfo Garcia Zamora hat die technischen Investitionen in den Bauernhöfen des mexikanischen Bundesstaates Zacatecas untersucht und dabei die von ehemaligen Migranten und von NichtMigranten geführten Betriebe unterschieden. Die ersten sind am häufigsten kapitalisiert (Lieferwagen, Traktoren, Schlepperpflüge, Hackpflüge usw.). Rodolfo Garcia Zamora, op. cit., S. 62, Tabelle 16.

34 Geneviève Cortes, op. cit.

35 Miguel Moctezuma Longoria, „La cultura migrante y el simbolismo de las remesas. Reflexiones a partir de la experiencia de Zacatecas“, in Raúl Delgado Wise und Beatrice Knerr (coord.), op. cit., S. 119. 
Der kollektive Migrant beteiligt sich an koordinierten Bemühungen, um seiner Herkunftsgemeinschaft zu helfen. In Mexiko und in El Salvador haben sich die Regierungen bemüht, bestimmte Geldflüsse umzuleiten und zu kanalisieren. Die Programme Dos por uno (Zwei für eins) und Tres por uno (Drei für eins) mündeten 1993 bzw. 1999 in Partnerschaften zwischen den Migrantenvereinen oder -clubs in den USA und ihren Heimatstaaten und -gemeinschaften (Dos por uno) sowie schliesslich mit dem Bundesstaat als drittem Partner ${ }^{36}$. Die Vereine zahlen einen bestimmten Betrag, die öffentliche Hand das Dreifache davon; die Mittel werden für den Bau oder die Wartung von Infrastrukturen - Wasserversorgungsnetze, Schulgebäude, Kirchen - verwendet. So kommen ansehnliche Summen zustande, die eine unverzichtbare Hilfe für die Subsistenz der lokalen Gebietskörperschaften bilden. Einige Autoren vertreten die Auffassung, dass man damit dem Staat die Investitionen teilweise abnehme, was zur Logik der vom Neoliberalismus befürworteten neuen Regierungsführung gehöre ${ }^{37}$. Das Modell wurde in anderen zentralamerikanischen Ländern übernommen, aber in Südamerika fasst es nur schwer Fuss.

Eine weitere Kritik an diesem Modell lautet, es habe kaum zur Schaffung von produktiven Unternehmen und zur Förderung der Investition und des Unternehmergeistes beigetragen ${ }^{38}$. Natürlich ist es einfacher, einen schönen öffentlichen Platz anzulegen, als Unternehmen zu gründen, obwohl das Potenzial dafür in mehreren Bereichen vorhanden ist ${ }^{39}$. Das Beispiel des Dorfes Atacheo de Regalado in Michoacan ist bezeichnend für die fehlende Managementfähigkeit. In diesem Dorf hatte der Priester mit dem Kapital von Migranten drei Projekte entwickelt: Herstellung von Musikinstrumenten für Musikensembles, eine Putenaufzucht und eine Ziegenaufzucht. Die drei Projekte erlitten Schiffbruch - allesamt wegen des schlechten Managements und der finanziellen Verluste (das Projekt der Putenaufzucht z.T. auch wegen einer Epidemie) $)^{40}$.

Zuerst muss das Humankapital ausgebildet werden, damit es das Finanzkapital zweckmässig nutzen und sich in die Produktionsketten integrieren kann. Auf kleinerem Massstab existieren solche Initiativen vor allem bei Produzenten, die sich auf die Herstellung von Lebensmitteln und anderen Produkten für die Emigrantengemeinschaften spezialisierten. In allen Städten der Vereinigten Staaten und Europas mit einer grossen Diaspora findet man Läden, die mexikanische,

36 Rafael Fernández de Castro, Rodolfo Garcia Zamora und Ana Vila Freyer (coord.), El programa 3x1 para migrantes. ¿Primera política transnacional en Mexico?, México, Universidad Autónoma de Zacatecas, 2006. Zuerst nannte sich das Programm Uno por uno: Der Migrantenverein und der Bundesstaat Zacatecas steuerten je einen Teil dazu bei. Es wurde sogar ein Versuch für Cuatro por uno mit der Beteiligung der Western Union unternommen (Western Union, Western Union Expands Initiative to Promote Community Economic Development in Mexico; Mexican State of Michoacan the Second State to benefit from 4x1 Program, Press Release, 23. Juni 2006, <http://ir.westernunion.com/ investor/releasedetail.cfm?ReleaseID=219081>).

37 Alejandro I. Canales, „Envois de fonds, développement et pauvreté, une perspective critique de l'Amérique latine“, in Isabel Yépez und Gioconda Herrera (dir.), op. cit., S. 195-217.

38 Mit dem Programm Tres por uno wurden zwischen 2002 und 2005 nur 4\% der gesamten Projekten als produktive Projekte im eigentlichen Sinne finanziert, der Rest floss in die Infrastrukturen (Rafael Fernández de Castro, Rodolfo Garcia Zamora und Ana Vila Freyer [coord.], op. cit., S. 17-18).

39 Alejandrino S. Loaiza, L. Herrea und César Sotomayor, op. cit.

40 Fernando Neira Orjuela, Sistematización de las experiencias en el uso productivo de remesas para el fomento del desarrollo: un estudio de caso en proyectos productivos del municipio de Atacheo de Regalado, en el Estado de Michoacán de Ocampo, México, México, UNAM y Grupo Chorlavi (Fondo Mink'a), 2008. 
peruanische, bolivianische Produkte usw. verkaufen. Auf dieser Grundlage lassen sich die Produktionstätigkeiten künftig sicherlich auf gehobenere Produkte mit höherer Wertschöpfung ausdehnen. Allerdings müssen dazu auch die Banken- und Finanznetze, die mit den Migranten und mit den Geldempfängern arbeiten, solider werden.

\section{Wirtschaftliche und soziale Auswirkungen der Geldsendungen}

Massive Geldsendungen an arme Gemeinschaften haben unterschiedliche soziale Auswirkungen - positive Aspekte für die Begünstigen, aber bisweilen „Störwirkungen“ für das soziale Umfeld. In den kleinen Gemeinschaften mit hoher Emigrationsrate (bisweilen über 50\% der Arbeitskräfte) sind die Folgen der Migration besonders sichtbar.

\section{Armutsverringerung}

Die Studien der Weltbank ${ }^{41}$ und anderer Institutionen zeigen, dass die Geldsendungen zu den ärmsten Bevölkerungsquintilen fliessen: „In Mexiko zum Beispiel sind die meisten Empfänger der Geldsendungen arm. 61\% der Haushalte, die Geld bekommen, gehören zum niedrigsten Einkommensquintil (Sendungen nicht mit gezählt), nur 4\% gehören zum höchsten Quintil.“42 Allgemein weisen die südamerikanischen Länder die gleiche ,,armenfreundliche“ Aufteilung auf. Eine Ausnahme bildet Peru, wo die Haushalte des niedrigsten Quintils 6\% der Heimatüberweisungen erhalten, das höchste Quintil jedoch $40 \%$ des Totals. Dieselbe ungleiche Verteilung ist in Nicaragua zu beobachten. In diesen beiden Ländern stammen die Migranten aus den wohlhabendsten Familien. In vier Ländern sind die Transfers mehr oder weniger gleichmässig auf reiche und arme Einkommensgruppen verteilt (Bolivien, Honduras, Dominikanische Republik, Haiti) ${ }^{43}$.

\section{Verringerung der Ungleichheit}

Die ,armutsbekämpfende“ Wirkung für die Geldempfänger ist erwiesen, aber mit der ungleichheitsverringernden Wirkung verhält es sich komplexer. Diese Wirkung muss vor dem globalen Hintergrund gesehen werden, denn zahlreiche Familien profitieren letztlich nicht von den Geldsendungen.

Die Untersuchung der Umverteilungswirkung der Geldtransfers auf die gesamte Einkommensumverteilung (einschliesslich der Transfers) zeigt, dass der Anteil der Ärmsten in allen Ländern stark abnimmt und dass bis auf Mexiko, Paraguay und El Salvador heute über die Hälfte der Geldempfänger zu den beiden höchsten Quintilen gehören; mit anderen Worten haben die Empfänger auf der Einkommensskala ihres Landes den Sprung nach oben geschafft. Das trifft besonders auf Länder zu, deren Emigranten mehrheitlich aus der höheren Schicht stammen (Peru). Selbst bei anfänglicher Einkommensgleichheit zwi-

41 Weltbank, The Development Impact of Workers'Remittances in Latin America, op . cit.

42 Pablo Fajnzylber und J. Humberto López, op. cit., S. 6 (Anm. d. Übers. : aus der französischen Übersetzung).

43 Ibid., S. 6. 
schen Migranten und Nicht-Migranten - wie in Sisid in Ecuador - verdienen die Migrantenfamilien plötzlich 50\% mehr als die Familien, die im Heimatland bleiben $^{44}$. Laut der Weltbank ist daraus zu schliessen, dass die Geldsendungen der Migranten zwar zweifellos einen Faktor für die Armutsverringerung bilden, für die Verringerung der Ungleichheit aber nicht die gleiche Rolle spielen, sondern den Status Quo eher verstärken oder erhalten ${ }^{45}$.

Diese auf makrosozialer Ebene grundlegenden globalen Feststellungen müssen je nach Land, Regionen und lokalen Verhältnissen nuanciert werden. Die Migrationsmodelle unterscheiden sich sehr stark nach Region, sozialer Schicht, Geschlecht, Land, Zweckbestimmung, Höhe der Überweisungen an die Familien und ihrer Verwendung. Die verfügbaren Studien zeigen, dass sich die Ungleichheit in Gebieten mit hoher Migration zuspitzt.

In Sisid in Ecuador z.B. werden die Bewohner neu in folgende Kategorien eingeteilt:

1. Empfänger von sehr hohen Beträgen (in der Regel bei Emigration des Ehepartners);

2. Empfänger von durchschnittlichen Beträgen (bei Emigration eines oder mehrerer Kinder) und nicht ausgewanderte „Fachleute“;

3. nicht ausgewanderte Familien;

4. Familien, die einen emigrierten Angehörigen, der sie unterstützt hat, verlieren und in äusserster Armut leben ${ }^{46}$.

In bestimmten Fällen bewirkt die Emigration vor Ort nur den Status Quo, weil die Emigranten nicht in ihrem Heimatdorf investieren, sondern in einer nahe gelegenen Stadt (das gilt z.B. für Bolivien) ${ }^{47}$. Darin äussert sich der Wille zum geplanten sozialen Aufstieg, der häufig mit der Suche nach besseren Schulen für die Ausbildung der Kinder einher geht.

\section{Arbeitsmarkt}

Die Migration von 20- bis 40-jährigen Männern verursacht einen Arbeitskräftemangel, der durch die Arbeit der Frauen oder durch neue Immigranten aus dem In- und Ausland nicht immer wettgemacht werden kann. Dies führt zur Einstellung landwirtschaftlicher Tätigkeiten und zu einer Konzentration in diesem Sektor, besonders in der Viehzucht. In anderen Bereichen, besonders in der Baubranche, steigt zudem das Arbeitsangebot, was jenes in der Landwirtschaft zusätzlich schmälert. So wird Boden aufgegeben oder an andere Eigentümer verpachtet und manche Grundstücke werden verkauft.

Die bisweilen erheblichen Geldsendungen der Migranten führen möglicherweise auch zu kürzeren Arbeitszeiten oder zu geringerer Beteiligung am Arbeitsmarkt. Die Umfragen von Pablo Fajnzylber und J. Humberto López zeigen, dass die

44 Centro de Desarrollo e Investigación Rural (CEDIR), Migración internacional en la comuna Sisid: cambios y adaptaciones en el territorio rural y economia local, Quito, CEDIR und Grupo Chorlavi (Fondo Mink'a), 2008, Abbildung 7.

45 Weltbank, The Development Impact of Workers' Remittances in Latin America, op. cit.

46 Centro de Desarrollo e Investigación Rural (CEDIR), op. cit.

47 Geneviève Cortes, op. cit. Roxana Dulón, op. cit. 
Anzahl Arbeitsstunden der Mitglieder von Migrantenfamilien in allen zehn Ländern der Stichprobe in der Stadt wie auf dem Land und für Frauen wie Männer abnimmt ${ }^{48}$. Die Beteiligung am Arbeitsmarkt geht ebenfalls zurück; Ausnahmen bilden Mexiko, Honduras, Guatemala und die Dominikanische Republik. Die Personengruppe, die mindestens vier Jahre die Grundschule besucht hat, weist die höchste Arbeitsmarktbeteiligung auf. Jim Airola führt die rückläufige Anzahl Arbeitsstunden in Mexiko auf die Geldsendungen zurück ${ }^{49}$. Nach Auffassung von Ezra Rosser kann sich der kontraproduktive Einkommenseffekt indessen positiv auswirken, wenn die Löhne in der Region steigen und einen Anreiz für Beschäftigung schaffen - vorausgesetzt, der Lohnzuwachs reicht aus, um das Plus an Freizeit, das die Migrantensendungen verschaffen, zu kompensieren $^{50}$. Mögliche soziale Folgen der Nichteinbindung in den Arbeitsmarkt bestehen auch in der Verschärfung der Gewalt unter jungen Arbeitslosen, welche Banden nach dem Vorbild der maras in Zentralamerika bilden ${ }^{51}$.

\section{Emigration und regionale Entwicklung}

Geldüberweisungen sollten im Grunde dazu beitragen, dass arme Bevölkerungen in ihrem Umfeld bleiben, doch die „Neureichen“ sind häufig darauf erpicht, ihr pueblo (Dorf) zu verlassen und in attraktivere Zentren der Region zu ziehen. Wie Béatrice Knerr zeigt, stammen die Migranten häufig aus Regionen mit besonders dürftigen Infrastrukturen und knappen Beschäftigungsmöglichkeiten. Wer im Land bleibt und arbeiten möchte, emigriert deshalb in die Hauptorte, was die Flucht aus den infolge der Migration praktisch verlassenen Dörfern noch verschärft. Hinsichtlich der Beschäftigung nennt die Autorin Beispiele für den „Ravenstein-Effekt“, d.h. die Verdrängung der Migranten durch Immigranten aus anderen Regionen und Ländern (z.B. aus der Ukraine nach Polen, aus Ägypten nach Jordanien oder aus Guatemala nach Chiapas in Mexiko ${ }^{52}$. Gleiches gilt für Ecuador, wo peruanische Landwirtschaftsarbeiter lokale Emigranten ablösen. Die „Rückkehrer" lassen sich auch lieber in wirtschaftlich und soziokulturell attraktiveren Orten nieder ${ }^{53}$. Die Gründe für den Wohnsitzwechsel hängen oft damit zusammen, dass die Migrantenfamilien den Kindern eine bessere Schulausbildung ermöglichen möchten und dass die häufig privaten weiterführenden Schulen sich in den Städten befinden.

Zur Ausbildung ist zu erwähnen, dass die Migrantengelder nur begrenzte Auswirkungen auf die Schulausbildung haben. Unter den Ärmsten hat die Zahl der Schulabbrecher abgenommen, aber Kinder von Migranten erzielen generell nicht unbedingt bessere Schulleistungen ${ }^{54}$.

48 Pablo Fajnzylber und J. Humberto López, op. cit., S. 31.

49 Jim Airola, op. cit.

50 Ezra Rosser, op. cit.

51 Ibid., S. 29.

52 Beatrice Knerr, „Dinàmicas económicas regionales frente a la migración laboral internacional. Reflexiones a partir de la experiencia de Zacatecas“, in Raúl Delgado Wise und Beatrice Knerr (coord.), op. cit., S. 137-170, S. 146. Die Theorie von Ernest George Ravenstein über die Substitution der Migrationen stammt bereits aus der Zeitperiode 1885-1890.

53 Ibid., S. 147.

54 Pablo Fajnzylber und J. Humberto López, op. cit. 
Die Kompensation der Arbeitsplatzverluste durch die Schaffung von Aktivitäten - dank den Migrantengeldern - beschränkt sich auf die lokale Nachfrage nach Verbrauchsgütern und, bei kollektiven Investitionen, auf den Bau von Infrastrukturen.

\section{Soziale Strukturen und Machtstrukturen}

$\mathrm{Zu}$ den Auswirkungen der Migration auf lokaler Ebene stellt sich ausserdem die folgende Frage: Können die ökonomischen Auswirkungen der Mittelüberweisungen die soziale Organisation der Empfängergemeinschaften in Frage stellen? Kommt es zum Bruch der Hierarchien und des traditionellen Solidaritätsgefüges oder sind die lokalen Gesellschaften in der Lage, die ökonomischen Veränderungen ohne soziale Umwälzungen zu verkraften?

Das zu knappe verfügbare Studienmaterial zeigt, dass die soziale Struktur der Dörfer mit hoher Migrationsrate durch das Geld, welches neue Machtgruppen schafft und die Gemeinschaften spaltet, allmählich untergraben wird; deshalb findet tatsächlich eine soziale Umbildung statt, die weitgehend vom fraglichen Land und vom Ausmass der Migration abhängt.

In den Staaten mit einer seit langem ethnisch stark durchmischten Bevölkerung (El Salvador, Dominikanische Republik, Mexiko) haben die Geldsendungen in erster Linie soziale Auswirkungen, weil sie den bislang verachteten sozialen Schichten zu einem gewissen empowerment verhelfen. Das Beispiel der dominikanischen „Kabarettkünstlerinnen“ in der Schweiz, die riesige Geldsummen an ihre Eltern schicken, ist bezeichnend für die soziale Aufwertung von Familien und Personen, die unten auf der sozialen Leiter stehen, aber wirtschaftlich erfolgreich sind ${ }^{55}$. Der moralische Aspekt spielt hier eine untergeordnete Rolle wahrscheinlich weil die dominikanische Gesellschaft bereits als ziemlich permissiv gilt.

In den Andenländern (Ecuador, Peru, Bolivien) scheint die soziale Ausgangsstruktur inflexibler und die hierarchischen Verhältnisse widersetzen sich Veränderungen. Dies gilt besonders für die Beziehung zwischen Frauen und Männern: Selbst wenn die Männer Tausende Kilometer weg arbeiten, überlassen sie die Entscheidungsbefugnis nicht den Frauen zu Hause, sondern wollen in kommunalen Angelegenheiten und im Familienleben das Sagen behalten ${ }^{56}$. In der Gemeinschaft von Sisid bewegen die autochthonen Einwohner die ExHacendados (Hacienda-Besitzer) dazu, kommunale Machtansprüche abzutreten, was einige Mestizen veranlasst hat, die Gemeinschaft völlig zu verlassen. Die Umwälzungen und der soziale Aufstieg hängen vom spezifischen Kontext und von der relativen Stärke der beteiligten Gruppen ab. Die Erhebungen sind nicht vollständig genug, um allgemeine Tendenzen in den Anden herauszuschälen.

Das Phänomen der Geldüberweisungen in Lateinamerika verursacht also in den ländlichen Gemeinschaften zweifellos eine tiefgreifende Umwälzung der sozialen Strukturen, die sich in Spannungen äussert, welche wiederum die politische

55 Jennifer Petree und Tahira Vargas, Dominicanos en Suiza. Pautas, practicas e impactos de la migración transnacional y el envió de remesas que vinculan Republica Dominicana y Suiza, EPFL, Cahiers du LaSUR, Nr. 8, Lausanne, Eidgenössische Technische Hochschule Lausanne, 2005

56 Die Geschlechterbeziehungen werden weiter unten erläutert. 
Macht am Ort und darüber hinaus beeinflussen. Dieses Phänomen geht einher mit anderen sozialen und kulturellen Schocks in den ländlichen Gegenden: Invasion des Tourismus, neue Anbautechniken (Beregnung) und Zuchttechniken (Weidemast) sowie bessere Infrastrukturen, die die Dörfer in die Nähe der Städte rücken.

Das Aufkommen der „Neureichen“ ist aber nicht mit weniger Ungleichheiten gleichzusetzen, weil parallel dazu ,neue Arme“ entstehen: In den Dörfern sind es die Ausgegrenzten der Migration oder Familien, die häufig durch einen Todesfall ihre Stütze im Ausland verloren haben. Die sozialen Strukturen formieren sich also in Regionen mit hoher Migrationsrate neu; auf Regionen, in denen fest etablierte Grossgrundbesitzer vorherrschen, die seit Jahrzehnten die Macht ausüben (z. B. in Chiapas oder in Oaxaca in Mexiko), trifft dies weniger zu. In den Städten verschwinden erfolgreiche Migranten in der dichten urbanen Masse, so dass keine wesentlichen Effekte spürbar sind.

\section{Geldüberweisungen und ethnische Zugehörigkeit}

Das Verhältnis zwischen ethnischen Faktoren und Migration wurde noch kaum untersucht. Eine Erhebung in einigen autochthonen Gemeinschaften mit hoher Emigrationsrate im Bundesstaat Oaxaca liess ersehen, dass die Migrantengelder kollektiv für die lokale Entwicklung verwendet werden, wobei die herkömmlichen kommunalen Kontrollmechanismen zum Tragen kommen. Der Migrant erachtet es als seine moralische und soziale Pflicht, zu öffentlichen Investitionen beizutragen, aber die Entscheidungen trifft nicht er, sondern die Kommunalversammlung. Die Migranten müssen theoretisch auch die Kommunalabgaben (verschiedene Arbeiten) mit bestreiten und auf Wunsch der Gemeinschaft ein Ädilamt bekleiden. Migranten, die zu weit weg wohnen, z.B. in Kalifornien, können einen Vertreter bezahlen, für dessen Handlungen sie dann allerdings haften $^{57}$. Bei Verstössen drohen strenge Strafen, die von der Bodenbeschlagnahme bis zum Entzug der gemeinschaftlichen Identität und zur Ausstossung reichen.

In den Anden ist eine Verdrängung der Mestizen-Eliten durch autochthone Migranten, die selbstbewusster und wohlhabender geworden sind, festzustellen. In Ecuador erwerben die autochthonen Bewohner immer grössere soziale Mobilität ${ }^{58}$. Die wenigen verfügbaren Studien zeigen allerdings nicht, ob die gemeinschaftlichen Traditionen dadurch gestärkt oder geschwächt werden. Die Mittelüberweisungen bleiben in allen Fällen und ungeachtet der ethnischen Zugehörigkeit grösstenteils bei den Familien. Die kollektive Kontrolle betrifft vor allem die zusätzlichen Mittel, die an die Gemeinschaften gehen. Dagegen kann man die Hypothese aufstellen, dass die wirtschaftlichen Veränderungen und der relative neue Reichtum bestimmter Familien in den autochthonen Gemeinschaften Spannungen verursachen, welche das gewohnte Führungssystem beeinträchtigen werden.

57 Beatriz Straffon, Paola Lopez und Anally Castellanos, „El Programa 3x1 para Migrantes en Oaxaca. Migracion indigena, practicas comunitarias y costumbres de participacion“, in Rafael Fernández de Castro, Rodolfo Garcia Zamora und Ana Vila Freyer (coord.), op. cit., S. 197-223.

58 Centro de Desarrollo e Investigación Rural (CEDIR), op. cit. Gloria Camacho und Kattya Hernández, op. cit. 
Migranten neigen bisweilen zu Luxus- und Prestigeausgaben, die aus der Perspektive der Entwicklung sinnlos, aber für den Erhalt des Sozialkapitals des Migranten durchaus sinnvoll erscheinen.

Unter dem Druck der Gemeinschaft oder aus freien Stücken versucht der Migrant (wie oben erwähnt), seine gesellschaftliche Position durch punktuelle Beiträge an bestimmte Werke oder Bauten zu verbessern, z.B. Kirchen, Schulen, Jahresfeste, und bringt bei seiner jährlichen Rückkehr Geschenke mit ${ }^{59}$. Besonderen Anklang findet der Bau eines Haus für die Familie, weil dies die Lebensbedingungen verbessert und wegen der Schaffung von symbolischem Kapital auch dem Prestigegedanken entspricht $t^{60}$. Das neue Heim muss bestimmte Kriterien erfüllen: Es soll modern sein, sich vom klassischen Stil der dörflichen Häuser abheben und wie die Musterhäuser in den Gastländern, in Immobilienzeitschriften oder Fernsehserien aussehen. Die Bauten der „Neureichen“ wirken deshalb oft extravagant, kitschig und nicht unbedingt funktionell. Die traditionelle Architektur und der Städtebau werden dadurch stark beeinträchtigt. In den Anden geht die schleichende Zersetzung des architektonischen Kolonialerbes nicht nur auf die Migration und auf Geschmacksverirrungen zurück, sondern auch auf das Fehlen einer Städteplanung.

Die Wirkung von Prunkausgaben darf indessen nicht übertrieben werden; Geldsendungen sind nicht mit Überfluss zu verwechseln und das Gros der Ausgaben ist für den täglichen Verbrauch bestimmt. Manuel Orozco stellt fest: „The cost of living in Latin American countries is at least two or three times the average amount received in remittances. " 61

\section{Unterschiede zwischen den Geschlechtern}

Wenn die Ehemänner das Land verlassen, übernehmen die Frauen Aufgaben, mit denen sie vorher nichts zu tun hatten, z.B. schwere Arbeit in der Landwirtschaft (Bodenbearbeitung) und in Geschäften, Aufgaben in der Kommunalpolitik usw. Dies führt häufig zur Überbelastung der Frauen, die nicht immer durch eine Familienhilfe oder durch Lohnarbeit wettgemacht werden kann.

Frauen sind oft zwischen neuen Anforderungen, die von ihnen Entscheidungsbefugnisse verlangen, und der Meinung ihrer Ehemänner, die per Telefon in Kontakt bleiben, hin und her gerissen. Eine Erhebung bei 229 Emigrierten aus der Stadt Jerez im mexikanischen Bundesstaat Zacatecas zeigte, dass Telefonkontakte sehr häufig sind: $50 \%$ der Migranten rufen mindestens ein Mal pro Woche an, $18 \%$ ein Mal alle zwei Wochen und unter $1 \%$ greift nie zum Telefon $^{62}$. In Sisid in Ecuador halten die Frauen während der Kommunalversammlung telefonisch die Verbindung zu ihren Männern in den Vereinigten Staaten, um für die Abstimmungen Weisungen zu bekommen ${ }^{63}$. In den Nachbarländern

59 Geneviève Cortes, op. cit.

60 Jennifer Petree und Tahira Vargas, op. cit.

61 Manuel Orozco, op. cit.

62 Siehe Rodolfo Garcia Zamora, op. cit., S. 159, Tabelle 8.

63 Roxana Dulón, op. cit. 
der Vereinigten Staaten, z.B. Mexiko, sind Rückreisen relativ häufig (in bestimmten Dörfern von Zacatecas einmal alle zwei Jahre $)^{64}$. Die gleiche Erhebung zeigt indessen auch, dass die Hälfte der Emigrierten überhaupt nicht zurückkehrt, sondern nur Geld schickt.

\section{Schlussfolgerung}

Die Migration zeitigt zwar auf lokaler und regionaler Ebene einige „kontraproduktive" oder Störwirkungen, denen indessen die folgenden positiven Aspekte die Waage halten: globale Verringerung der Armut in Gemeinschaften mit starker Emigration, Verbesserung des Komforts der Haushalte, bessere Hygiene- und Gesundheitsbedingungen, relative Kapitalisierung der Landwirtschaftsbetriebe (Maschinen, Brunnen, Transportmittel) und Vergrösserung der Besitztümer.

Die Geldsendungen der Migration tragen zur Vorsorge und zur sozialen Sicherheit bei, welche der Staat in Lateinamerika nicht leisten kann. Die Altersvorsorge der Eltern wird durch die Migration der Kinder und der Eltern selbst gewährleistet. Die ,transnationale Familie“ agiert auf allen Stufen, um das Überleben der heutigen und der nächsten Generationen zu sichern. Genau diese Tatsache verringert jedoch den Druck auf den Staat und bremst die Entwicklung von Sozialsystemen, die auf Besteuerung und sozialer Ersparnis beruhen. Der Begriff ,transnationale Familie“ ist ambivalent. Er bezeichnet ein etabliertes, relativ solides Globalisierungs-Modell innerhalb und zwischen Staaten, die jedoch selbst nicht transnational sind, weil sie ihre Sozialpolitiken meistens nicht koordinieren. Für illegale Migranten kommt erschwerend hinzu, dass sie nicht in den Sozialhilfe- oder Rentensystemen erfasst sind.

Es wäre verfehlt, zu behaupten, dass die Migrationsbewegungen von Arbeitnehmern aus dem Süden in den Norden für die in der Heimat gebliebenen Familienmitglieder einen Segen oder ein Geschenk des Himmels darstellten. Die Trennung der Familien verursacht in den Herkunftsgemeinschaften mitunter gravierende Probleme. Besonders wenn die Männer mehrere Monate oder sogar Jahre lang weg bleiben, müssen die Frauen mit grosser Mühe für die Familie sorgen, auf dem Feld arbeiten und sich am Gemeindeleben beteiligen. Folgen dieser Überbelastung sind das Verlassen der Familie, Trennungen und Scheidungen oder die Zusammenführung der Ehepartner im Ausland, wobei das Problem der Erziehung und der emotionalen Fürsorge für die Kinder gänzlich den Grosseltern, Tanten und Onkeln im Herkunftsland überlassen wird.

Die Migration in Lateinamerika wird von den internationalen und nationalen Organisationen trotzdem als positiv beurteilt, weil sie Bewegung in Gesellschaften bringt, die bislang wegen fehlender Mittel in einem Zustand der Unterwerfung und der Lähmung verharrten. Deswegen soll man die ,Tatsache der Migration und die Gelder aus der Migration“ nicht ablehnen, sondern die beiden Bewegungen so gestalten, dass sie den Urhebern und den Empfängern sowie ihren Gemeinschaften bestmöglich nutzen. Die Staaten und die internationalen Organisationen sollten aufgefordert werden, sich als Entwicklungspartner an diesem Spiel zu beteiligen und neben Finanzhilfe auch Fachwissen beizusteuern

64 Volker Hamman, „Diferencias intraregionales en la zona de alta migración zacatecana“, in Raúl Delgado Wise und Beatrice Knerr (coord.), op. cit., S. 193-206, S. 199, Tabelle 4. 
(uno, dos, tres, cuatro por uno). So könnten insbesondere MikrokreditProgramme mit der Mobilisierung der Migrationsgelder verknüpft werden.

Die Migration zeigt, dass die „Selbsthilfe der Armen“ auf persönlicher und familiärer Ebene weit besser klappt, als wenn die Reichen sich einmischen. Die privaten Geldüberweisungen der Migranten übersteigen bekanntlich die Auslandsdirektinvestitionen. Dies bedeutet nicht, dass diese Gelder an die Stelle des staatlichen Handels und der Aussenhilfe treten sollen, aber es wird klar, dass in der Gleichung der Entwicklung neue Partner - die Migranten - berücksichtigt und gänzlich in die Reflexionsprozesse und in die Projektumsetzung integriert werden müssen.

\section{Bibliographie}

Airola, Jim, „Labor Supply in Response to Remittance Income: The Case of Mexico“, The Journal of Developing Areas, Bd. 41, Nr. 2, Spring 2008.

Airola, Jim, „The Use of Remittance Income in Mexico (Report)“, International Migration Review, Bd. 41, Nr. 4, 2007.

Amuedo-Dorantes, Catalina and Susan Pozo, „On the Use of Differing Money Transmission Methods by Mexican Immigrants“, International Migration Review, Bd. 39, Nr. 3, 2005.

Auroi, Claude, „Cambios en la pequeña agricultura andina: hacia la intensificación. Una encuesta a tres comunidades en el Departamento de Cusco, Perú“, Congreso CEISAL Bratislava, 5-7 de julio de 2004 (inédit).

Auroi, Claude, „Situación alimenticia en América Latina y futuro de la producción agropecuaria para pequeños campesinos“, Colloque CEISAL, avril 2007, Bruxelles (à paraître en 2008).

Auroi, Claude et Jean-Luc Maurer, Tradition et modernisation des économies rurales: Asie-AfriqueAmérique latine. Mélanges en l'honneur de Gilbert Etienne, Paris, Presses Universitaires de France, 1998, $394 \mathrm{~S}$.

Auroi, Claude et Isabel Yépez del Castillo (dir.), Economie solidaire et commerce équitable: acteurs et actrices d'Europe et d'Amérique latine, Genève, Institut universitaire d'études du développement (IUED); Louvain, Presses Universitaires de Louvain, 2006.

Auroi, Claude, „Les envois de fonds pour le développement local. Réflexions à partir de cas latinoaméricains“, in Isabel Yépez et Gioconda Herrera (dir.), Nouvelles migrations latino-américaines en Europe, Barcelona, Publicacions i Edicions de la Universitat de Barcelona, S. 169-194.

Banco Central de Reserva de El Salvador, Metodología de calculo e importancia de las remesas familiares en El Salvador, 2004, <http://www.cemla-remesas.org/PDF/seminariomx/0510MX-ES-PPTElSalvador.pdf $>$.

Bendixen and Associates, Remittances and the Dominican Republic: Survey of Recipients in the Dominican Republic, Survey of Senders in the United States, November 2004, <http://www.iadb.org/NEWS/ docs/SurveysRemittanceFlows_e.ppt>.

Centro de Desarrollo e Investigación Rural (CEDIR), Migración internacional en la comuna Sisid: cambios y adaptaciones en el territorio rural y economia local, Quito, CEDIR y Grupo Chorlavi (Fondo Mink'a), 2008.

Camacho, Gloria y Kattya Hernández, Territorios entrelazados: Impactos de la emigracion internacional para el desarrollo rural de Suscal, Quito, Instituto de estudios ecuatorianos, Grupo Chorlavi, 2008.

Canales, Alejandro I., „Envois de fonds, développement et pauvreté, une perspective critique de l'Amérique latine", in Isabel Yépez et Gioconda Herrera (dir.), Nouvelles migrations latino-américaines en Europe, Barcelona, Publicacions i Edicions de la Universitat de Barcelona, S. 195-217.

Cortes, Geneviève, Partir pour rester: survie et mutation des sociétés paysannes (Bolivie), coll. „A travers champs“, Paris, Institut de recherche sur le développement (IRD), 2000.

Delgado Wise, Raúl y Beatrice Knerr (coord.), Contribuciones al análisis de la migración internacional y el desarrollo regional en México, Universidad Autónoma de Zacatecas, 2005.

Dulón, Roxana, Migración transnacional de bolivianos y bolivianas a la Argentina y su impacto en comunidades de origen, Sucre, Fundación Pasos y Grupo Chorlavi (Fondo Mink'a), 2008.

Durand, Jorge and Douglas S. Massey, „Mexican Migration to the US : A Critical Review“, Latin American Research Review, Bd. 27, Nr. 2, 1992, S. 3-43. 
Faret, Laurent, „Les impacts socioculturels de la circulation migratoire dans les environnements d'origine: le cas du Mexique“, in Eric Guerassimov (dir.), Migrations internationales, mobilités et développement, Paris, L'Harmattan, 2005, S. 273-311.

Fajnzylber, Pablo and J. Humberto López, Close to Home: The Development Impact of Remittances in Latin America, Conference Edition, Washington, DC, The World Bank, 2007.

Fischer Peter A., Reiner Martin and Thomas Straubhaar, „Interdependencies between Development and Migration“, in Thomas Hammar, Grete Brochmann, Kristof Tamas and Thomas Faist (eds.), International Migration, Immobility and Development, Oxford, Berg, 1997, S. 91-132.

Fernández de Castro, Rafael, Rodolfo Garcia Zamora y Ana Vila Freyer (coord.), El programa 3x1 para migrantes. ¿Primera política transnacional en Mexico?, México, Universidad Autónoma de Zacatecas, 2006.

Garcia Zamora, Rodolfo, Migración, remesas y desarrollo, los retos de las organizaciones migrantes mexicanas en Estados Unidos, México, Universidad Autónoma de Zacatecas, 2005.

Gosh, Bimal, Migrants' Remittances and Development: Myths, Rhetoric and Realities, Geneva, International Organization for Migration (IOM), 2006.

Hamman, Volker, „Diferencias intraregionales en la zona de alta migración zacatecana“, in Raúl Delgado Wise y Beatrice Knerr (coord.), Contribuciones al análisis de la migración internacional y el desarrollo regional en México, Universidad Autónoma de Zacatecas, 2005, S. 193-206.

Hamann, Volker, The Impact of International Labor Migration on Regional Development: The Example of Zacatecas, Mexico; Kassel, Kassel University Press, 2006.

Hammar, Thomas, Grete Brochmann, Kristof Tamas and Thomas Faist (eds.), International Migration, Immobility and Development, Oxford, Berg, 1997.

Hermele, Kenneth, „The Discourse on Migration and Development“, in Thomas Hammar, Grete Brochmann, Kristof Tamas and Thomas Faist (eds.), International Migration, Immobility and Development, Oxford, Berg, 1997, S. 133-158.

Hernandez-Coss, Raúl, Lessons from the US-Mexico Remittance Corridor on Shifting from Informal to Formal Transfer System, World Bank Working Paper, Nr. 47, Washington, DC, World Bank, 2005.

International Monetary Fund (IMF), „Workers' Remittances and Economic Development“, chapter 2, World Economic Outlook: Globalisation and External Imbalances, Washington, DC, IMF, 2005, $<$ http://www.imf.org/external>.

Instituto Nacional de Estadisticas, Geografia e Informatica (INEGI), Encuesta Nacional de Ingresos y Gastos de Hogares, Mexico, 2000.

Inter-American Development Bank (IDB), Enviando Dinero a Casa: Marcador de la Industria de Remesas, Washington, DC, IDB 2006.

Inter-American Development Bank (IDB), Remittance Flows to Latin America and the Caribbean, 2004, Washington, DC, IDB, 2004.

Inter-American Development Bank (IDB), Remittance Senders and Receivers: Tracking the Transnational Channels, Washington, DC, IDB, 2003.

Inter-American Development Bank (IDB), Remittances 2005: Promoting Financial Democracy, Washington, DC, IDB, 2006.

Inter-American Development Bank (IDB), Sending Money Home: Remittance Recipients in the Dominican Republic and Remittance Senders from the United States, New York, IDB and Columbia University, 2004.

Inter-American Development Bank (IDB), State by State Survey of Remittance Senders: United States to Latin America, Washington, DC, IDB, 2004.

Inter-American Development Bank (IDB), Survey of Remittance Senders: United States to Latin America, Washington, DC, IDB, 2002.

Inter-American Dialogue (IAD), Making the Most of Family Remittances, Second Report of the InterAmerican Dialogue Task Force on Remittances, Washington, DC, IAD, 2007.

Knerr, Beatrice, „Dinàmicas económicas regionales frente a la migración laboral internacional. Teorías y experiencias globales“, in Raúl Delgado Wise y Beatrice Knerr (coord.), Contribuciones al análisis de la migración internacional y el desarrollo regional en México, Universidad Autónoma de Zacatecas, 2005, S. 137-170.

Loaiza, Alejandrino S., L. Herrea y César Sotomayor, Las Remesas de los Migrantes Quillabambinos en Italia y sus Implicancias en el Desarrollo Local, Cusco, Proyecto de Desarrollo Corredor Puno-Cusco y Grupo Chorlavi, 2008.

Lozano Ascencio, Fernando, „De excluidos sociales a heroes sexenales. Discurso oficial y remesas en Mexico“, in Raúl Delgado Wise y Beatrice Knerr (coord.), Contribuciones al análisis de la migración internacional y el desarrollo regional en México, Universidad Autónoma de Zacatecas, 2005, S. 41-65. 
Neira Orjuela, Fernando, Sistematización de las experiencias en el uso productivo de remesas para el fomento del desarrollo: un estudio de caso en proyectos productivos del municipio de Atacheo de Regalado, en el Estado de Michoacán de Ocampo, México, México, UNAM y Grupo Chorlavi (Fondi Mink'a), 2008.

Organisation de coopération et de développement économiques (OCDE), Migration et développement, un nouveau partenariat pour la coopération, compte rendu de conférence, Paris, OCDE, 2008.

Orozco, Manuel, Transnational Families: Lives on the Edge, but in Pursuit of Change, 2006, zitiert von Ezra Rosser, Remittances, College of Law Research Paper, Nr. 40, Washington, DC, American University College of Law, 2008, <http://ssrn.com/abstract=1024177>.

Osorio Perez, Flor, W. Mejia, G. Restrepo, A. Milena y Y. Cifuentes, De Productor de Café a Pueblo Emigrante, Bogotà, Universidad Javeriana, Universidad tecnologica de Pereira y Grupo Chorlavi (Fondo Mink'a), 2008.

Petree, Jennifer y Tahira Vargas, Dominicanos en Suiza. Pautas, prácticas e impactos de la migración transnacional y el envió de remesas que vinculan Republica Dominicana y Suiza, EPFL, Cahiers du LaSUR, Nr. 8, Lausanne, Eidgenössische Technische Hochschule Lausanne, 2005.

Rosser, Ezra, Remittances, College of Law Research Paper, Nr. 40, Washington, DC, American University College of Law, 2008, <http://ssrn.com/abstract=1024177>.

Straffon, Beatriz, Paola Lopez y Anally Castellanos, „El Programa 3x1 para Migrantes en Oaxaca. Migracion indigena, practicas comunitarias y costumbres de participacion“, in Rafael Fernández de Castro, Rodolfo Garcia Zamora y Ana Vila Freyer (coord.), El programa 3x1 para migrantes. ¿Primera política transnacional en Mexico?, México, Universidad Autónoma de Zacatecas, 2006, S. 197-223.

Suki, Leonora, Financial Institutions and the Remittance Market in the Domican Republic, New York, Center on Globalization and Sustainable Development, The Earth Institute at Columbia University, 2004.

Western Union, Western Union Expands Initiative to Promote Community Economic Development in Mexico; Mexican State of Michoacan the Second State to benefit from 4x1 Program, Press Release, 23 June 2006, <http://ir.westernunion.com/investor/releasedetail.cfm?ReleaseID=219081>.

Wilson, John F., Progress Report on CEMLA Remittances Compilation Manual for LAC Countries, verfügbar über Internet, „Remittances Statistics: First Meeting of the Luxemburg Group June 2006“, $<$ http://www.imf.org/external/np/sta/bop/2006/luxgrp/060106.htm>.

World Bank, Global Economic Prospects 2006: Economic Implications of Remittances and Migration, Press launch, with Christopher Neal, François Bourguignon, Hans Timmer, Dilip Ratha, William Shaw, Washington DC, The World Bank, 16 November 2005.

World Bank, Global Economic Prospects 2006: Economic Implications of Remittances and Migration, Washington, DC, World Bank, 2006.

World Bank, The Development Impact of Workers' Remittances in Latin America, Report, Nr. 37026, Washington, DC, World Bank, 2006.

Wucker, Michele, „Remittances, the Perpetual Migration Machine“, World Policy Journal, Bd. 21, Nr. 2, Summer 2004.

Yépez del Castillo, Isabel et Gioconda Herrera Mosquera (dir.), Nouvelles migrations latino-américaines en Europe, Barcelona, Publicacions i Edicions de la Universitat de Barcelona, 2008. 